\title{
ANÁLISE DA DEFORMAÇÃO RESIDUAL LONGITUDINAL E SUA RELAÇÃO COM PROPRIEDADES TECNOLÓGICAS DA MADEIRA DE ACÁCIA-NEGRA ${ }^{1}$
}

Rafael de Avila Delucis ${ }^{2}$, Rafael Beltrame ${ }^{3}$, Darci Alberto Gatto ${ }^{3}$, Pedro Henrique Gonzalez de Cademartori ${ }^{4}$ e Eduardo Schneid ${ }^{5}$

\begin{abstract}
RESUMO - A madeira de acácia-negra tem sido utilizada em nichos que conferem pouco valor agregado à esse material. Diante disso, este trabalho teve como objetivo analisar as deformações residuais longitudinais (DRL) e suas relações com propriedades da madeira de Acacia mearnsii. Para tanto, foram selecionadas 60 árvores em plantios com idades distintas (4 e 7 anos) no Município de Piratini, RS. As características de crescimento avaliadas consistiram em diâmetro à altura do peito (1,3 m; DAP), espessura de casca (EP) e altura comercial (h). As propriedades físicas avaliadas massa específica básica $(\rho)$ e teorde umidade à base seca (Tu). Já o tempo de propagação de onda ultrassonora foi determinado em conformidade com o procedimento NBR 15521. As propriedades mecânicas foram o módulo de elasticidade dinâmico (Ed), bem como as propriedades descritas no procedimento normativo D143-94, através dos ensaios de flexão estática, compressão paralela às fibras e Dureza Janka. A DRL e suas relações com as propriedades da madeira mostraram-se semelhantes aos resultados encontrados na literatura para a madeira de espécies do gênero Eucalyptus. No entanto, em magnitude, tais níveis se mostraram sensivelmente menores, conferindo à madeira de acácia-negra no que se refere a esse parâmetro de qualidade, indicação para a confecção de produtos sólidos.
\end{abstract}

Palavras-chave: CIRAD-Forêt; Tensões de crescimento; Qualidade da madeira.

\section{ANALYSIS OF LONGITUDINAL RESIDUAL STRAIN AND ITS RELATION TO TECHNOLOGICAL PROPERTIES OF BLACK WATTLE WOOD}

\begin{abstract}
The black wattle wood has been used in niches that confer low value to this material. Therefore, this study aimed to analyze the longitudinal residual strain (LRS) and its relations with Acacia mearnsii de wild wood properties. To achieve this, 60 trees with 4 and 7 years were selected from a forest population located in Piratini, RS. Diameter at breast height (1.3 m; DBH), bark thickness (BT) and commercial height (h) were evaluated. The physical properties evaluated were specific gravity $(\rho)$ and moisture content on dry basis (Tu). The propagation time of ultrasonic wave was determined as described in NBR 15521 and then the dynamic modulus of elasticity (Ed) was measured. The mechanical properties was evaluated according to D143-94 standard by static bending, parallel compression to fibers and Janka hardness tests. The LRS and its relations with the wood properties were similar to the results found in the literature for wood from Eucalyptus species. However, in magnitude, these levels were sensibly lower, giving black wattle indication to produce solid wood products, when it comes to this quality parameter.
\end{abstract}

Keywords: CIRAD-Forêt; Growth stresses; Wood quality.

\footnotetext{
${ }^{1}$ Recebido em 13.06.2013 aceito para publicação em 25.03.2015.

${ }^{2}$ Universidade Federal de Pelotas, Programa de Pós-Graduação em Ciência e Engenharia de Materiais, Pelotas, Rio Grande do Sul, Brasil. E-mail: <r.delucis@hotmail.com>.

${ }^{3}$ Universidade Federal de Pelotas, Centro de Engenharias, Pelotas, Rio Grande do Sul, Brasil. E-mail: $<$ beltrame.rafael@yahoo.com.br $>$ e $<$ darcigatto@yahoo.com $>$.

${ }^{4}$ Universidade Federal do Paraná, Programa de Pós-Graduação em Engenharia Florestal, Curitiba, Paraná, Brasil. E-mail: <pedrocademartori@gmai.com>.

${ }^{5}$ Universidade Federal de Santa Catarina, Programa de Pós-Graduação em Engenharia Civil, Florianópolis, Santa Catarina, Brasil. E-mail: <eduardoschneid87@gmail.com>.
} 


\section{INTRODUÇÃO}

Entre as espécies arbóreas mais cultivadas no país, estima-se que os plantios de Acacia mearsii De Wild (acácia-negra) ultrapassaram em 2011 os 146 mil hectares, e cerca de 40 mil famílias retiram seu sustento de atividades ligadas à sua utilização (ABRAF, 2012; MÜLLER, 2006).

Nos dias atuais, a utilização da madeira encontra-se condicionada à caracterização e melhoramento de sua qualidade. Nesse contexto, um dos principais objetivos das pesquisas do setor se trata da previsão e contenção de custos decorrentes de seu processamento. Dessa maneira, torna-se de estratégica importância a definição das matérias-primas mais adequadas, em conformidade com o produto final requerido.

Em consideração às peculiaridades intrínsecas aos produtos madeireiros, entre as principais causas de desperdícios, os efeitos causados pelas tensões de crescimento configuram-se como um dos maiores empecilhos à produção de madeira serrada.

Os defeitos decorrentes da liberação das tensões de crescimento no tecido lenhoso de árvores são as fendas e rachaduras nos extremos das toras, os empenamentos e rachaduras longitudinais durante o desdobro de toras e pranchões e o cerne quebradiço, devido à excessiva tensão de compressão nas proximidades da medula (NICHOLSON, 1973). Contudo, as tensões de crescimento desempenham papel de grande importância no desenvolvimento de árvores vivas. Van Vik (1978) enunciou essa importância na sustentação dos indivíduos arbóreos quando em seu crescimento, relatando que propiciam a rigidez estrutural demandada pela ação de ventos fortes e pelo peso de sua copa, bem como por inclinações topográficas.

Para avaliação indireta das tensões de crescimento longitudinais, entre a gama de métodos não destrutivos, os mais mencionados na literatura são o uso do Extensômetro (CIRAD-Fôret), o Strain Gauge e o Stress Wave Timer, sendo o extensômetro (CIRAD-Forêt) o mais utilizado por características como a versatilidade e a simplicidade na obtenção dos dados.

Por meio de artigos já publicados no meio científico, verifica-se que o estudo das tensões de crescimento já galgou degraus importantes, a fim de que o conhecimento acerca da caracterização do comportamento da madeira sob sua influência seja consolidado. Apesar disso, ainda carece de muitas outras elucidações, como por exemplo, em consideração à espécies alternativas às do gênero Eucalyptus.

Este estudo teve em vista analisar as deformações residuais longitudinais, bem como verificar suas relações com algumas características de crescimento e propriedades físicas e mecânicas da madeira de acácianegra.

\section{MATERIAL E MÉTODOS}

\section{1. Área de estudo}

Os povoamentos de acácia-negra utilizados neste estudo localizavam-se em propriedades de pequenos produtores florestais do município de Piratini, Rio grande do Sul (31³0'09"S 5305'12"O), e foram implantados em espaçamento inicial de 1,5 x 3,0 m, dos quais foram selecionadas 30 árvores, de acordo as idades de plantio de 4 e 7 anos. Tais árvores foram selecionadas em razão do seu estado fitossanitário, fuste retilíneo e tronco cilíndrico, evitando-se árvores de bordadura e em terreno com presença de inclinação acentuada.

\subsection{Avaliação da Deformação Residual Longitudinal (DRL)}

Por meio de um extensômetro produzido pelo Centre de Cooperation Internationale em Recherche Agronomique Pour Le Développement - CIRAD-Fôret, realizou-se a mensuração da DRL, de forma indireta, em árvores em pé e vivas em quatro posições (direções) ao redor do tronco. A fim de padronizar essas leituras, a escolha das posições ocorreu conforme a orientação dos pontos cardeais, coincidentes com as linhas e entre linhas do plantio.

Inicialmente, realizou-se o descascamento da árvore de forma a abrir-se uma janela ao redor da árvore na altura do diâmetro à altura do peito (1,3 m; DAP). Com o auxílio de um gabarito, foram inseridos dois pinos distantes entre si, verticalmente, de 0,045 m, na direção da grã, tendo esses por finalidade a fixação do aparelho de medição que consistiu, basicamente, de um relógio comparador digital. A posteriori, utilizando uma furadeira manual, incidiu-se uma broca de $0,02 \mathrm{~m}$ de diâmetro entre os pinos, a uma profundidade de 0,02 m. A anotação realizada consistiu no valor mostrado pelo visor digital do extensômetro após o rompimento do tecido lenhoso (com cuidado para não realizar o procedimento na presença de ventos). 


\subsection{Características de Crescimento}

Após a avaliação da DRL, o DAP foi mensurado utilizando uma suta com resolução de $0,001 \mathrm{~m}$. Em seguida, aproveitando o descascamento parcial da árvore, foi possível medir-se a espessura da casca com o auxílio de um paquímetro digital com resolução de 0,01 mm.

Para a medição da altura, foram abatidas cinco árvores de cada idade entre as selecionadas para a avaliação da DRL. Por intermédio de fita métrica com resolução de $0,001 \mathrm{~m}$, determinou-se a altura comercial (altura até a primeira bifurcação) de cada indivíduo posto sobre o solo.

\subsection{Propriedades Físicas da Madeira}

Por meio de um machado, retirou-se uma amostra do alburno sem casca de cerca de $100 \mathrm{~cm}^{3}$ de cada árvore viva na altura do DAP. As amostras foram embaladas em um filme plástico, para evitar troca de umidade com o ambiente externo. Subsequentemente, tais amostras foram destinadas ao Laboratório de Anatomia da Madeira do Curso de Engenharia Industrial Madeireira da Universidade Federal de Pelotas (UFPEL).

Após, foram pesadas para a obtenção do teor de umidade natural (Tu). Posteriormente, o material foi encharcado em água por imersão simples e seco em estufa laboratorial sob a temperatura de $100 \pm 3^{\circ} \mathrm{C}$, sendo ambos os procedimentos (saturação e secagem) até que as amostras obtivessem massa constante. Para a medição do volume saturado (método do deslocamento pelo empuxo) e da massa seca, utilizou-se uma balança analítica com resolução de 0,01 g. Com esses dados, foi calculada a massa específica básica $(\rho)$, conforme o procedimento D143-94 da American Society for Testingand Materials (ASTM, 2007).

\subsection{Ensaios Mecânicos}

Para tal, foram utilizadas as 10 árvores abatidas para o dimensionamento da altura. As amostras foram segmentadas da primeira tora de $1,5 \mathrm{~m}$ de comprimento. Dessa forma, realizou-se o desdobro primário, e de cada prancha de $0,05 \mathrm{~m}$ de espessura, foram retiradas amostras orientadas em relação aos planos anatômicos, rejeitando-se a casca e a região da medula. As dimensões físicas das amostras foram de 2 × 2 × $30 \mathrm{~cm}$ no ensaio de flexão estática; 5 × 5 × 20 cm no ensaio de compressão paralela às fibras; e $5 \times 5 \times 15 \mathrm{~cm}$ no ensaio de Dureza Janka.



As amostras confeccionadas passaram por um período de acondicionamento na câmara climatizada do Curso de Engenharia Industrial Madeireira da UFPel, câmara essa que impôs ao material a condição de $65 \%$ de umidade relativa do ar e $20^{\circ} \mathrm{C}$ de temperatura. As amostras permaneceram até obter o teor de umidade de equilíbrio respectivo à sua condição no interior da câmara (massa constante), e o referido teor de umidade foi de aproximadamente $12 \%$.

Por meio da utilização de um aparelho de ultrassom da marca Proceq S.A., origem Suíça, em cada uma das amostras foi avaliado o tempo de propagação de onda ultrassonora na direção longitudinal, conforme o procedimento NBR 15521, da Associação Brasileira de Normas Técnicas - ABNT (ABNT, 2007). Com os dados referentes a esse ensaio, foram calculados a velocidade ultrassonora (Eq. 1) e o módulo de elasticidade dinâmico (Eq. 2) de cada amostra.

$$
\begin{gathered}
\mathrm{v}=\frac{\mathrm{d}}{\mathrm{t}} \\
\mathrm{Ed}=\mathrm{v}^{2} \cdot \rho_{\mathrm{a} 12 \%} \cdot 10^{-6}
\end{gathered}
$$

em que $\mathrm{v}=$ velocidade ultrassonora $\left(\mathrm{m} \cdot \mathrm{s}^{-1}\right) ; \mathrm{d}=$ comprimento da amostra $(\mathrm{m}) ; \mathrm{t}=$ tempo de propagação da onda ultrassonora (s); Ed = módulo de elasticidade estático $(\mathrm{MPa}) ;$ e $\rho_{\mathrm{a} 12 \%=}$ massa específica aparente a $12 \%\left(\mathrm{~kg} \cdot \mathrm{m}^{-3}\right)$.

Nas amostras de flexão estática, foram utilizados dois transdutores piezoelétricos exponenciais de 45 $\mathrm{kHz}$ de frequência, com a finalidade de emitir a onda ultrassonora e, após a sua propagação no material, recebê-la.

Para as amostras de compressão paralela às fibras, utilizaram-se transdutores de face plana de $54 \mathrm{kHz}$ de frequência. Nessa avaliação, para garantir que na interface entre a madeira e o transdutor não houvessem impurezas que pudessem interferir na medição, foi aplicada uma fina camada de vaselina farmacêutica na seção transversal das amostras.

As propriedades mecânicas foram avaliadas conforme o procedimento adaptado D143-94 da ASTM (ASTM, 2007), por meio de ensaios de flexão estática, compressão paralela às fibras e dureza Janka. Para cada ensaio, foram utilizadas 60 amostras.

Na realização dos ensaios mecânicos, utilizou-se uma máquina universal de ensaios mecânicos

Revista Árvore, Viçosa-MG, v.39, n.3, p.585-594, 2015 
eletromecânica informativa (EMIC) com capacidade para $300 \mathrm{kN}$ e extensômetro para a medida das deformações no ensaio de compressão paralela às fibras.

Posteriormente à realização dos ensaios mecânicos, as amostras foram submetidas à secagem convencional, por meio de uma estufa laboratorial com temperatura de $102 \pm 3{ }^{\circ} \mathrm{C}$ até atingirem teor de umidade de $0 \%$; em seguida, foi mensurada a massa anidra de cada amostra, a fim de calcular o teor de umidade no momento do ensaio, utilizando-se a equação 2 . Por fim, foi corrigido o valor da resistência da madeira para o teor de umidadepadrão $(12 \%)$, conforme a proposta do procedimento NBR 7190 da ABNT (ABNT, 1997).

\subsection{Análise Estatística}

Para o delineamento experimental, os dados da DRL foram considerados inteiramente casualizados no esquema fatorial, tendo como fatores a posição ao redor do tronco em quatro níveis e a idade dos povoamentos em dois níveis. Os fatores tidos como significativos pelo teste $\mathrm{F}$ foram desdobrados $\mathrm{e}$ analisados separadamente pelo teste LSD de Fisher. Com a aplicação do teste de homogeneidade de variância de Levene, verificou-se a necessidade de transformação dessa variável antes da aplicação da ANOVA, a qual foi realizada pela aplicação da função matemática $1 / x$.

As demais propriedades avaliadas foram comparadas entre idades por análises de variância (ANOVA) e, dada a diferença significativa dos valores, foram realizados testes de média LSD de Fisher em 1 e 5\% de significância. Analogamente à variável DRL, o teste de homogeneidade de variância de Levene indicou que as variáveis $\mathrm{f}_{\mathrm{c} 0} \mathrm{e} \mathrm{E}_{\mathrm{c} 0}$ necessitavam de transformação, a qual foi realizada para ambas as variáveis, conforme a função matemática $x^{3}$.

A fim de explicar a relação funcional da DRL com as demais variáveis avaliadas, foram realizadas análises de correlação simples (Pearson).

\section{RESULTADOS}

Por meio da análise de variância fatorial realizada, verificou-se diferença significativa entre os valores médios de DRL, na comparação entre idades ( $\mathrm{F}=$ $16,90 ; p<0,01)$ e entre posições $(F=6,87 ; p<0,01)$. A interação entre esses dois fatores (idade e posição), da mesma forma, denotou-se significativa $(F=6,60$; $\mathrm{p}<0,01)$.

Revista Árvore, Viçosa-MG, v.39, n.3, p.585-594, 2015
Sem distinção entre idades, o valor médio da deformação residual longitudinal denotou-se $0,066 \mathrm{~mm}$. Quando comparados entre idades, esses valores médios diferiram estatisticamente, conforme pode ser visualizado pelas letras de testes de médias na Tabela 1 e na ilustração da Figura 1.

No que tange, portanto, a intensidade das deformações residuais longitudinais, o povoamento de 7 anos possivelmente apresentará menor incidência de defeitos em decorrência dos mecanismos que permeiam as tensões de crescimento.

Pela Tabela 1, também é possível verificar que, de forma significativa, os valores médios de DRL variaram entre as idades de cada posição, exceto na posição Leste e entre posições, de forma que, como ilustra a Figura 1, a posição Sul apresentou valores sensivelmente mais elevados. Dessa forma, pela variabilidade denotada nos valores quando comparados ao redor do tronco, para a avaliação da DRL utilizando a metodologia proposta neste estudo, é necessário que sejam efetuadas leituras no mínimo em quatro posições ao redor do tronco.

Pela Tabela 2, na comparação entre as idades, verificou-se significativa variação nos valores médios de massa específica básica e de DAP, no sentido de que ambos os parâmetros se denotaram mais elevados no povoamento mais antigo (7 anos) deste estudo.

Pela Tabela 2, verificou-se que o valor médio do teor de umidade $(\mathrm{Tu})$ foi significativamente maior no povoamento de 4 anos. Nesse sentido, os demais parâmetros avaliados foram mais elevados em termos de suas médias aritméticas para a idade de 7 anos. Em destaque, verificou-se notória diferença nos valores de DAP, na comparação entre idades, variação que foi de 31,3 em valores porcentuais.

Os parâmetros avaliados pelos ensaios mecânicos destrutivos e pelo ensaio não destrutivo (ultrassom) são apresentados a seguir, em termos de sua estatística descritiva e dos testes de médias realizados (Tabela 3 ).

É possível observar pela Tabela 3 que as variáveis $\mathrm{E}_{\mathrm{M}}, \mathrm{E}_{\mathrm{c} 0}, \mathrm{f}_{\mathrm{c} 0} \mathrm{e} \mathrm{Ed}_{\mathrm{c}}$ diferiram quando comparadas entre idades, de forma que em todas essas o povoamento de 7 anos apresentou valores mais elevados. Quanto às relações entre a DRL e todas essas variáveis, os resultados obtidos pela análise de correlação simples (Pearson) são apresentados na Tabela 4. 
Em consideração às características de crescimento, na Tabela 4 se pode observar que a DRL apresentou correlação significativa e negativa com o DAP. Dessa forma, pode-se verificar a tendência de declínio dos valores de DRLà medida que aumentam os valores de DAP. Visto que os valores de DAP lograram a maior diferença comparada entre idades, sendo possível verificar que o desenvolvimento das árvores mitigou os níveis de DRL.
Os parâmetros EP e Tu apresentaram-se significativa e positivamente correlacionados com a DRL no povoamento de 4 anos. No povoamento de 7 anos, além do DAP, o EP denotou-se correlacionado de forma significativa com a DRL. Porém, a $\rho$ não apresentou correlações significativas com a DRL, para os povoamentos de ambas as idades.

Tabela 1 - Valores médios de DRL em razão das idades e orientações de plantio. Table 1 - Average values of LRS as a function of age and planting orientation.

\begin{tabular}{|c|c|c|c|c|c|}
\hline & Norte & Sul & Leste & Oeste & $\mu$ \\
\hline 4 anos & $0,071_{(19,53)} \mathrm{Bb}$ & $0,085_{(14,40)} \mathrm{Cb}$ & $0,062_{(20,14)} \mathrm{Aa}$ & $0,063_{(12,51)} \mathrm{ABb}$ & $0,071_{(9,76)} \mathrm{b}$ \\
\hline 7 anos & $0,055_{(49,12)}^{(19,3)} \mathrm{Aa}$ & $0,072{ }_{(33,53)}^{(14,40)} \mathrm{Ba}$ & $0,068_{(23,41)}^{(20,14)} \mathrm{Ba}$ & $0,056_{(18,62)} \mathrm{Ba}$ & $0,062_{(18,27)}^{\mathrm{a}}$ \\
\hline$\mu$ & $0,063_{(67,79)} \mathrm{A}$ & $0,078_{(42,23)} \mathrm{C}$ & $0,065_{(28,64)} \mathrm{BC}$ & $0,059_{(23,99)} \mathrm{B}$ & 0,066 \\
\hline
\end{tabular}

Em que $\mu=$ média ( $\mathrm{mm}$ ). Médias seguidas por letras diferentes (maiúsculas na linha e minúsculas na coluna) possuem diferença estatisticamente significativa entre si, de acordo com o teste LSD de Fisher. Coeficiente de variação entre parênteses (\%).



7 anos

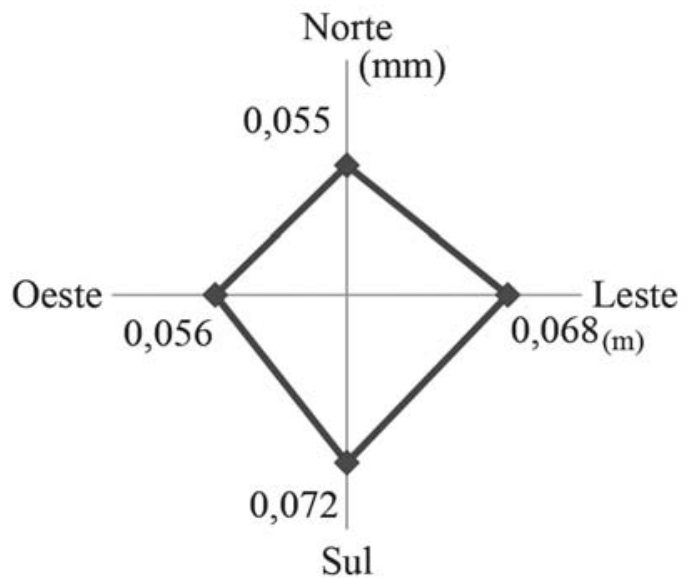

Figura 1 - Variabilidade dos valores médios de DRL ao redor do tronco das árvores de acácia-negra nas idades de 4 e 7 anos. Figure 1 - Variation of LRS average values around the trunk of black wattle trees for four and seven years.

Tabela 2 - Características de crescimento e propriedades físicas.

Table 2 - Growth characteristics and physical properties.

\begin{tabular}{|c|c|c|c|c|c|}
\hline & $\begin{array}{l}\text { DAP } \\
(\mathrm{cm})\end{array}$ & $\begin{array}{c}\mathrm{H} \\
(\mathrm{m})\end{array}$ & $\begin{array}{c}\mathrm{EP} \\
(\mathrm{mm})\end{array}$ & $\begin{array}{c}\rho \\
\left(\mathrm{g} \cdot \mathrm{cm}^{-3}\right)\end{array}$ & $\begin{array}{l}\mathrm{Tu} \\
(\%)\end{array}$ \\
\hline 4 anos & $13,10_{(5,87)} \mathrm{a}$ & $13,94_{(4,93)} \mathrm{a}$ & $4,59_{(14,44)} \mathrm{a}$ & $0,55_{(5,82)} \mathrm{a}$ & $77,71_{(20,62)} b$ \\
\hline 7 anos & $17,10_{(17,91)} \mathrm{b}$ & $17,74_{(5,59)} \mathrm{b}$ & $5,88_{(12,88)} b$ & $0,67_{(5,04)}^{b}$ & $64,28_{(24,61)} a$ \\
\hline Valor F & $33,34 * *$ & $24,89 * *$ & $24,87 * *$ & $114,01 * *$ & $7,2 * *$ \\
\hline
\end{tabular}

Em que DAP = diâmetro à altura do peito $(1,3 \mathrm{~m}) ; \mathrm{H}=$ altura comercial; $\mathrm{EP}=$ espessura de casca; $\rho=$ massa específica básica; Tu= teor de umidade; $\mu=$ média; $* *=$ significativo em $1 \%$ de probabilidade de erro. Médias seguidas por letras diferentes em uma mesma coluna possuem diferença estatisticamente significativa de acordo com o teste LSD de Fisher. Coeficiente de variação entre parênteses (\%). 
Tabela 3 - Valores médios das propriedades mecânicas.

Table 3 - Average values for the mechanical properties.

\begin{tabular}{|c|c|c|c|c|c|c|c|c|}
\hline & $\begin{array}{c}\mathrm{E}_{\mathrm{M}} \\
(\mathrm{MPa})\end{array}$ & $\begin{array}{c}\mathrm{f}_{\mathrm{M}} \\
(\mathrm{MPa})\end{array}$ & $\begin{array}{c}\mathrm{E}_{\mathrm{co}} \\
(\mathrm{MPa})\end{array}$ & $\begin{array}{c}\mathrm{f}_{\mathrm{c} 0} \\
(\mathrm{MPa})\end{array}$ & $\begin{array}{c}\mathrm{f}_{\mathrm{HR}} \\
(\mathrm{MPa})\end{array}$ & $\begin{array}{c}\mathrm{f}_{\mathrm{HT}} \\
(\mathrm{MPa})\end{array}$ & $\begin{array}{c}\mathrm{Ed}_{\mathrm{M}} \\
(\mathrm{MPa})\end{array}$ & $\begin{array}{c}\mathrm{Ed}_{\mathrm{c}} \\
(\mathrm{MPa})\end{array}$ \\
\hline \multicolumn{9}{|c|}{4 anos } \\
\hline$\mu$ & $8313 \mathrm{a}$ & 56,19 a & $12237 \mathrm{a}$ & $38,21 \mathrm{a}$ & $35,23 \mathrm{a}$ & 36,86 a & $12621 \mathrm{a}$ & $17491 \mathrm{a}$ \\
\hline $\mathrm{CV}$ & 29,95 & 46,01 & 23,18 & $\begin{array}{l}9,83 \\
7 \text { anos }\end{array}$ & 22,55 & 20,79 & 22,54 & 11,8 \\
\hline$\mu$ & 8369 a & 59,85 a & $13859 \mathrm{~b}$ & $39,87 \mathrm{~b}$ & 39,49 a & $37,61 \mathrm{a}$ & $14159 \mathrm{a}$ & $18484 \mathrm{~b}$ \\
\hline $\mathrm{CV}$ & 25,97 & 42,35 & 18,13 & 11,26 & 18,88 & 34,13 & 22,08 & 6,92 \\
\hline Teste F & $4,27 *$ & $0,69^{\mathrm{ns}}$ & $6,52 *$ & $7,08 *$ & $2,28^{\mathrm{ns}}$ & $0,37^{\mathrm{ns}}$ & $3,83^{\text {ns }}$ & $4,24 *$ \\
\hline
\end{tabular}

Em que $\mathrm{f}_{\mathrm{M}}=$ módulo de ruptura $; \mathrm{E}_{\mathrm{M}}=$ módulo de elasticidade estático; $\mathrm{f}_{\mathrm{c} 0}=$ resistência à compressão paralela às fibras; $\mathrm{E}_{\mathrm{c} 0}=$ módulo de elasticidade; $\mathrm{f}_{\mathrm{uR}}=$ resistência à dureza Janka radial; $\mathrm{f}_{\mathrm{HT}}=$ resistência a dureza Janka tangencial; Ed $\mathrm{d}_{\mathrm{M}}=$ Módulo de elasticidade dinâmico de flexão estática; $\mathrm{Ed}=$ Módulo de elasticidade dinâmico de compressão paralela às fibras; $\mu=$ média; $\mathrm{CV}=$ coeficiente de variação (\%). Médias seguidas por letras diferentes em uma mesma coluna possuem diferença estatisticamente significativa de acordo com o teste LSD de Fisher.

Tabela 4 - Correlação entre a DRL, as características de crescimento e propriedades físicas e mecânicas.

Table 4 - Correlation between DRL, growth characteristics and physical and mechanical properties.

\begin{tabular}{lcc}
\hline \multirow{2}{*}{ Propriedade } & \multicolumn{2}{c}{$\mathrm{r}$} \\
\cline { 2 - 3 } & 4 anos & 7 anos \\
\hline DAP & $-0,65^{* *}$ & $-0,48^{* *}$ \\
$\mathrm{H}$ & $0,01^{\mathrm{ns}}$ & $0,29^{\mathrm{ns}}$ \\
$\mathrm{EP}$ & $0,40^{*}$ & $0,41^{*}$ \\
$\mathrm{Tu}$ & $0,40^{*}$ & $0,21^{\mathrm{ns}}$ \\
$\rho$ & $-0,24^{\mathrm{ns}}$ & $-0,21^{\mathrm{ns}}$ \\
$\mathrm{f}_{\mathrm{M}}$ & $0,18^{\mathrm{ns}}$ & $-0,42^{*}$ \\
$\mathrm{E}_{\mathrm{M}}$ & $-0,14^{\mathrm{ns}}$ & $-0,09^{\text {ns }}$ \\
$\mathrm{f}_{\mathrm{co}}$ & $-0,28^{\mathrm{ns}}$ & $0,23^{\mathrm{ns}}$ \\
$\mathrm{E}_{\mathrm{c} 0}$ & $-0,21^{\mathrm{ns}}$ & $-0,54^{* *}$ \\
$\mathrm{f}_{\mathrm{HR}}$ & $0,19^{\mathrm{ns}}$ & $-0,21^{\mathrm{ns}}$ \\
$\mathrm{f}_{\mathrm{HT}}$ & $0,35^{\mathrm{ns}}$ & $-0,33^{\mathrm{ns}}$ \\
$\mathrm{Ed}_{\mathrm{M}}$ & $-0,16^{\mathrm{ns}}$ & $-0,18^{\mathrm{ns}}$ \\
$\mathrm{Ed}_{\mathrm{c}}$ & $0,13^{\mathrm{ns}}$ & $-0,41^{*}$ \\
\hline
\end{tabular}

Em que $\mathrm{DAP}=$ diâmetro à altura do peito $(1,3 \mathrm{~m}) ; \mathrm{H}=$ altura comercial; $\mathrm{EP}=$ espessura de casca; $\rho=$ massa específica básica; $\mathrm{Tu}=$ teor de umidade; $\mathrm{f}_{\mathrm{M}}=$ Módulo de ruptura da flexão estática; $\mathrm{E}_{\mathrm{M}}=$ Módulo de elasticidade da flexão estática; $\mathrm{f}_{\mathrm{c} 0}=$ Resistência à compressão paralela às fibras; $\mathrm{E}=$ Módulo de elasticidade da compressão paralela às fibras; $\mathrm{f}_{\mathrm{HR}}=$ Dureza Janka radial; $\mathrm{f}_{\mathrm{HT}}=$ Dureza Janka tangencial; $\mathrm{Ed}_{\mathrm{M}}$ = Módulo de elasticidade dinâmico de flexão estática; $\mathrm{Ed}_{\mathrm{c}}=$ Módulo de elasticidade dinâmico de compressão paralela às fibras; $r={ }^{c}$ coeficiente correlação com a DRL; $*=5 \%$ de nível de probabilidade de erro; $* *=1 \%$ de nível de probabilidade ${ }^{\text {ns }}=$ estatisticamente não significativo.

Em consideração às propriedades mecânicas, em um contexto geral, verifica-se na Tabela 4 que as correlações respectivas ao povoamento mais velho (7 anos) denotaram-se melhores, em comparação com as do povoamento mais novo (4 anos), inferindo, assim, maior homogeneidade em suas propriedades.

Tal resultado tem, possivelmente, base na proporção entre os lenhos juvenil e adulto. Dessa forma, por apresentar maior quantidade de lenho juvenil, é pertinente presumir que o povoamento mais jovem é caracterizado pelas propriedades bastante heterogêneas que, como é de consenso, são intrínsecas ao lenho juvenil.

No ensaio de compressão paralela às fibras, a variável $\mathrm{E}_{\mathrm{c} 0}$ apresentou correlação significativa e negativa. Portanto, à medida que os valores de DRL aumentam, essa variável tende a sofrer declínio de forma proporcional.

\section{DISCUSSÃO}

Para comparação dos resultados obtidos neste estudo com os demais trabalhos científicos, foram utilizados estudos respectivos à madeira de Eucalyptus spp., devido à escassez de subsídios literários relacionados a esse tema, para a madeira de Acacia mearnsii.

Genericamente, os valores médios encontrados na literatura são sensivelmente mais elevados em povoamentos de Eucalyptus spp. com idades semelhantes. Muneri (2000), avaliando árvores de Eucalyptus cloeziana com 4 anos, relatou valores da DRL entre 0,038 e 0,132 mm, com valor médio de 0,077 mm. Já Pádua et al. (2004), examinando clones de 4 e 7 anos de Eucalyptus sp., verificaram valores médios de 0,074 e $0,083 \mathrm{~mm}$, respectivamente, e Trugilho et al. (2007), em povoamentos de Eucalyptus sp. de 6 anos, obtiveram valores de 0,088 $\mathrm{mm}$.

Com relação a outras espécies de folhosas, Watanabe et al. (2012), avaliando a madeira de cedro-japonês (Cryptomeria japônica) aos 16 anos de idade, proveniente do Japão, reportaram valor médio de DRL de 0,061 mm. A partir desse valor médio, o qual pode ser considerado

Revista Árvore, Viçosa-MG, v.39, n.3, p.585-594, 2015 
semelhante ao deste estudo $(0,066 \mathrm{~mm})$, esses autores avaliaram que a madeira de seu estudo possui potencial para ser utilizada como matéria-prima para a confecção de produtos sólidos. Já Clair et al. (2003), avaliando a madeira de castanheira (Castanea sativa) oriunda da França, obtiveram valores de DRL entre 0,090 e 0,300 $\mathrm{mm}$. Ambos os trabalhos citados indicam que os níveis de DRL deste estudo estão dentro dos limites evidenciados em espécies de folhosas, tanto no meio científico nacional, quanto internacional.

A diferença significativa entre as idades avaliadas foi - de forma semelhante - evidenciada por Cardoso Jr. et al. (2005), que, tomando como parâmetro a variação da idade (3, 5, 7, 8 e 9 anos) de clones híbridos do gênero Eucalyptus, concluíram que o valor médio de DRL variou de forma desuniforme. Portanto, conforme este estudo, o maior valor médio ocorreu aos 7 anos $(0,066 \mathrm{~mm})$ e o menor, aos 3 anos $(0,054 \mathrm{~mm})$.

Para Muneri et al. (2000), que avaliaram povoamentos de Eucalyptus cloeziana aos 4 anos de idade, a direção Sul apresentou valores mais elevados. No entanto, em povoamentos mais velhos (10 anos), segundo Muneri et al. (1999), a direção Oeste mostrou valores mais elevados. Assim como esses autores, Trugilho e Oliveira (2008), avaliando as variações das diferentes idades (8, 13, 15 e 19 anos) em árvores de Eucalyptus dunnii, identificaram tendência de aumento dos valores médios de DRL com o aumento da idade.

A diferença significativa da DRL encontrada entre as posições de leitura diverge dos resultados obtidos por Lima et al. (2004), que não verificaram diferença significativa ao redor do tronco. Outros autores, por meio de análises estatísticas, verificaram significância avaliando a diferença entre os valores médios de DRL ao longo da circunferência do tronco em árvores de Eucalyptus spp. (MUNERI et al., 1999; MUNERI et al., 2000; BELTRAME et al., 2012).

Segundo Beltrame et al. (2012), os valores médios de DRL são significativamente diferentes quando comparados com as direções de plantio. Nesse contexto, conforme o estudo desses autores, os valores médios demonstraram-se significativamente mais elevados para o sentido Leste.

Alguns autores afirmaram que os níveis de tensão de crescimento são bastante influenciados pela proporção de lenho juvenil, da massa específica, do diâmetro e da proporção de lenho de reação (BENDTSEN, 1978;
KELLISON, 1981). Dessa forma, os autores inferiram que há tendência inversa à proporcionalidade entre a idade e a DRL, embora não tenham analisado essas variáveis.

Quanto aos valores médios obtidos das propriedades biométricas das árvores, eles se mostraram semelhantes ao encontrado na literatura para características de crescimento e propriedades físicas da madeira de acácia-negra (FREDO et al., 1999; RAWCHAL, 2001).

Muneri et al. (2000) encontraram correlação positiva e significativa da DRL com a massa específica do alburno $(\mathrm{r}=0,40)$, com o DAP $(\mathrm{r}=-0,51)$ e com a altura $(\mathrm{r}=$ -0,44) de árvores de Eucalyptus cloeziana. Segundo Beltrame et al. (2012), o DAP apresentou correlação com a DRL $(r=-0,27)$, bem como a espessura de casca $(\mathrm{r}=0,24)$.

Em comparação com as correlações obtidas por esses autores, pode-se observar que as correlações verificadas neste trabalho foram maiores em termos dos coeficientes de correlação visualizados na Tabela 4 , destacando-se o DAP $(\mathrm{r}=-0,65$ e $-0,48)$ e o EP (r $=0,41$ e 0,40$)$.

Carvalho et al. (2010), classificando povoamentos de Eucalyptus urophylla e Corymbia citriodora, segundo cinco classes diamétricas, observaram correlações negativas e significativas mais elevadas entre DAP e DRL $(r=-0,84)$.

Conforme Tolfo (2003), o diâmetro é um dos parâmetros mais representativos a ser tomado em consideração quando na seleção precoce de árvores com vistas ao aumento da qualidade da madeira.

Visto que o ciclo de vida de povoamentos de acácia-negra é bastante curto (cerca de 10 anos), é possível presumir que, embora não tenha sido avaliada a proporção de madeira adulta, provavelmente essa proporção é maior proporção nos povoamentos mais velhos avaliados no presente estudo (7 anos), comparando-os com os mais jovens (4 anos). Segundo Vidaurre et al. (2011), a madeira juvenil caracterizase pela variação ostensiva de algumas propriedades anatômicas e físicas, conforme a espécie e as condições ambientais. Assim como neste estudo para o fM do povoamento de 4 anos e para $\mathrm{E}_{\mathrm{M}}$ do povoamento de 7 anos, Lima et al. (2004) não verificaram correlação significativa entre as variáveis avaliadas pelo ensaio de flexão estática.

Revista Árvore, Viçosa-MG, v.39, n.3, p.585-594, 2015

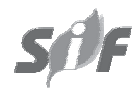


Fang et al. (2008) atribuíram os desacordos existentes na literatura relacionados a essas correlações às variações no ângulo microfibrilar da camada $S_{2}$ da parede secundária das fibras causada pelas tensões de crescimento. Ainda conforme esses autores, alguns modelos matemáticos, utilizando como variável independente o ângulo microfibrilar, têm confirmado essa hipótese.

Assim como pode ser visualizado na Tabela 4 sobre o povoamento de 7 anos, Raymond et al. (2004) verificaram que ocorreu em povoamentos de Eucalyptus globulus correlação significativa da DRL com a variável $\mathrm{f}_{\mathrm{M}}$; tais autores avaliaram que o $\mathrm{E}_{\mathrm{M}}$ também apresentou significativa correlação com a variável DRL.

Lima et al. (2004) concluíram que não houve correlação significativa entre a DRL e os parâmetros respectivos desse ensaio mecânico. No entanto, Fang et al. (2008), em povoamentos de álamo (Populus deltoides), verificaram correlações negativas e significativas do módulo de elasticidade e de ruptura no ensaio de compressão paralela às fibras com a DRL.

Essa tendência inversamente proporcional da resistência à compressão pode ser atribuída ao menor teor de lignina entre os caracteres anatômicos da madeira que causa, em fibras não lignificadas, planos de deslizamento nos espaços intercelulares e achatamento das fibras, resultando em fibras mais esbeltas (FANG et al., 2008).

Assim como na maioria dos parâmetros avaliados pelos ensaios mecânicos de flexão estática e de compressão paralela às fibras, os parâmetros avaliados pelo ensaio mecânico de Dureza Janka, quando correlacionados com a DRL, não apresentaram correlação significativa. De forma semelhante, Lima et al. (2004) não encontraram correlação significativa entre tais variáveis com a DRL.

\section{CONCLUSÕES}

Os níveis verificados de DRL apresentaram relações semelhantes com as propriedades da madeira, na comparação com trabalhos científicos publicados examinando madeiras do gênero Eucalyptus. Nesse contexto, denotaram-se sensivelmente menos elevados. Dessa forma, a madeira de Acacia mearnsii, no tocante à intensidade dos valores de DRL, pode ser indicada para a confecção de produtos sólidos.
O povoamento mais velho (7 anos) apresentou valor médio de DRL mais adequado. Além do fator idade, o fator posição apresentou efeito significativo na variação da DRL. Dessa forma, na posição Sul foram mensurados os níveis mais elevados de DRL em ambas as idades. Pela variabilidade entre as posições mesuradas, conclui-se que são necessárias quatro posições de leitura.

Constatou-se que as características de crescimento apresentaram melhores correlações com a DRL do que as propriedades físicas e mecânicas, com destaque para o DAP e o EP.

\section{REFERÊNCIAS}

ASSOCIAÇÃO BRASILEIRA DE PRODUTORES DE FLORESTAS PLANTADAS - ABRAF. Anuário estatístico da ABRAF 2012, ano base 2011. Brasília: 2012. 150p.

\section{ASSOCIAÇÃO BRASILEIRA DE NORMAS} TÉCNICAS - ABNT. Projetos de estruturas de madeira - NBR 7190. Rio de Janeiro: 1997. 107p.

\section{ASSOCIAÇÃO BRASILEIRA DE NORMAS TÉCNICAS - ABNT. Ensaios não destrutivos - Ultra-som - Classificação mecânica de madeira serrada de dicotiledôneas - NBR 15521. Rio de Janeiro: 2007. 7p.}

\section{AMERICAN SOCIETY FOR TESTING AND MATERIALS - ASTM. Standard Test methods for small clear specimens of timber - ASTM D143-94. West Conshohocken: 2007. 32p.}

BENDTSEN, B.A. Properties of wood from improved and intensively maneged trees. Forest Products Journal, v.28, n.10, p.61-71, 1978.

BELTRAME, R.; LAZAROTTO, M.; HASELEIN, C.R.; SANTINI, E.J.; SCHNEIDER, P. R.; AGUIAR, A.M. Determinação das deformações residuais longitudinais decorrentes das tensões de crescimento em Eucalyptus spp. Ciência Florestal, v.22, n.2, p.343-351, 2012.

CARDOSO JR, A.A; TRUGILHO, P.F.; LIMA, J.T.; ROSADO, S.C.S.; MENDES, L.M.

Deformação residual longitudinal em diferentes 
espaçamentos e idades em clone de híbrido de Eucalyptus. Cerne, v.11, p.218-224, 2005.

CARVALHO, A.M.; GONÇALVES, M.P.M.; AMPARADO, K.F.; LATORRACA, J.V.F.; GARCIA, R.A. Correlações da altura e diâmetro com as tensões de crescimento em árvores de Corymbia citriodora e Eucalyptus urophylla. Revista Árvore, v.34, n.2, p.323-331, 2010.

CLAIR, B.; RUELLE, J.; THIBAUT, B. Relationship between growth stresses, mechanic-physical properties and proportion of fibre with gelatinous layer in chestnut (Castanea sativa Mill.).

Holzforschung, v. 57, n.2, p.189-195, 2003.

FANG, C.H.; GUIBAL, D.; CLAIR, B.; GRIL, J.; LIU, Y.M.; LIU, S.Q. Relationships between growth stress and wood properties in Poplar 2 I69 (Populus deltoids Bartr. cv."Lux" ex I-69/55).

Annals of Forest Sciences, v.65, n.3, p.307, 2008.

FREDO, A.; FOELKEL, C.E.B.; FRIZZO, S.M.B.; SILVA, M.C.M. Elementos minerais em madeiras de eucaliptos e acácia-negra e sua influência na indústria de celulose kraft branqueada. Ciência Florestal, v.9, n.1, p.193-209, 1999.

KELLISON, R. C. Characteristics affecting quality of timber from plantations, their determination and scope for modification. In: IUFRO WORLD CONGRESS 17., 1981, Kyoto. Proceedings... Kyoto: 1981. p.77-87. *LIMA, J.T.; TRUGILHO, P.F.; ROSADO, S.C.S.; CRUZ, C.R.. Deformações residuais longitudinais decorrentes de tensões de crescimento em eucaliptos e suas associações com outras propriedades. Revista Árvore, v.28, n.1, p. 107-116, 2004.

MÜller, I. Avaliação da produtividade de Acácia mearnsii De Wild. (Acácia negra) em função de diferentes espaçamentos. 2006. 133f. Dissertação (Mestrado em Engenharia de Produção) Faculdade de Ciências Agrárias e Veterinárias, Universidade Federal de Santa Maria, Santa Maria, 2006.

TOLFO, A.L.T. Estudos da viabilidade de aplicação da seleção precoce em testes clonais de Eucalyptus spp. e qualidade da madeira para polpa celulósica. 2003. 50f. Dissertação (Mestrado em Genética e Melhoramento de Plantas) - Faculdade de Ciências Agrárias e Veterinárias, Universidade Estadual Paulista, Jaboticabal, 2003.

MUNERI, A.; LEGATE, W.; PALMER, G. Relationships between surface growth strain and some tree wood and sawn timber characteristics of Eucalyptus cloeziana. Southern African Forestry Journal, v. 187, n.1, p.41-49, 1999.

MUNERI, A.; KNIGHT, J.; LEGGATE, W.; PALMER, G. Relationships between surface longitudinal growth strain and tree size, wood properties and timber distortion of 4 year old plantation grown Eucalyptus cloeziana. In: IUFRO CONFERENCE -THE FUTURE OF EUCALYPTS FOR WOOD PRODUCTS, 2000, Launceston. 2000. IUFRO Proceedings... Launceston: 2000. p.292-300.

NICHOLSON, J.E. Growth stresses differences in Eucalyptus. Forest Science, v.9, n.3, p.169, 1973.

PÁDUA, F.A.; TRUGILHO, P.F.; ROSADO, S.C.S.; LIMA, J.T.; CARDOSO JR, A.A.; OLIVEIRA, A.C. Estimativas de parâmetros genéticos das tensões de crescimento em clones de Eucalyptus.

Scientia Forestalis, v.50, n.66, p.152-160, 2004.

RAWCHAL, M.F.G.; DEDECEK, R.A.; CURCIO, G.R.; SIMON, A.A. Produção de madeira, Casca e Tanino de Acacia mearnsii com Sete Anos, em Solos Derivados de Micaxistos e Arenito no Município de Piratini, RS. Colombo: Embrapa Florestas, 2001.4p. (Embrapa Florestas. Comunicado Técnico, 54).

RAYMOND, C.A.; KUBEA, P.D.; PINKARDA, L.; SAVAGEA, L.; BRADLEYA, A.D. Evaluation of non-destructive methods of measuring growth stress in Eucalyptus globulus: relationships between strain wood properties and stress. Forest Ecologyand Management. v.190, n.2-3, p.187-200, 2004.

TRUGILHO, P.F.; ROSADO, S.C.S.; LIMA, J.T.; PÁDUA, F.A.; SOUZA, M.A.M. Deformação residual longitudinal (DRL) e sua relação com as

Revista Árvore, Viçosa-MG, v.39, n.3, p.585-594, 2015 
características de crescimento da árvore em clones de Eucalyptus. Cerne, v.13, n.2, p. 130137, 2007.

TRUGILHO, P.F.; OLIVEIRA, J.T.S. Relationships and estimates of longitudinal growth stress in Eucalyptus dunnii at different ages. Revista Árvore, v.32, n.4, p.723-729, 2008.

van WYK, J.L. Hardwood sawmilling can have a bright future in South Africa. South African Forestry Journal, v.107, n.1, p.47-58, 1978.
VIDAURRE, G.; LOMBARDI, L.R.; OLIVEIRA, J.T.S.; ARANTES, M.D.C. Lenho Juvenil e Adulto e as Propriedades da Madeira.

Floresta e Ambiente, v.18, n.4, p.469-480, 2011.

WATANABE, K.; YAMASHITA, K.; NOSHIRO, S. Non-destructive evaluation of surface longitudinal growth strainon Sugi (Cryptomeria japonica) green logs using near-infrared spectroscopy.

Journal of wood Science. v.58, n.3, p.267272, 2012.

Revista Árvore, Viçosa-MG, v.39, n.3, p.585-594, 2015 